Polymer Journal, Vol. 9, No. 6, pp 527-535 (1977)

\title{
Characterization and Solution Properties of Randomly Branched Polymers
}

\author{
Hideomi Matsuda, Izumi Yamada, Masaru Okabe, \\ and Shigetaka KuroIwA \\ Department of Chemical Engineering, Faculty of Textile Science, \\ Shinshu University, Ueda 386, Japan.
}

(Received November 18, 1976)

\begin{abstract}
The characterization method of branched polymers recently proposed by the present authors was tested experimentally by using three samples of randomly branched polystyrene. The distribution curve of the molecular weight and the correlation between branching parameter and molecular weight were determined from the sedimentation velocity and diffusion patterns of each polymer sample in a theta solvent, cyclohexane at $35^{\circ} \mathrm{C}$, and it was ascertained that the average molecular weights derived from the distribution curve were in good agreement with the results of osmotic pressure and light scattering measurements. The molecular weight distribution curve thus established was utilized for converting the sedimentation pattern into the sedimentation constant vs. molecular weight relationship of each polymer, from which an average density of branch points was evaluated with the aid of the Kurata-Fukatsu theory. This estimate of the average branch-point density was of reasonable magnitude.

KEY WORDS Branched Polymer / Molecular Weight Distribution /

Polymer Characterization / Sedimentation / Diffusion / Solution

Properties / Kurata-Fukatsu Theory /
\end{abstract}

Since Benoit proposed a universal calibration method for the gel permeation chromatography (GPC), ${ }^{1}$ GPC has been applied to the characterization of branched polymers, ${ }^{2-4}$ and experimental studies of branched polymers have been rapidly developed. It is difficult, however, in GPC measurements to reproduce a constant experimental condition, and laborious to establish the universal calibration curve. Moreover, in order to apply the GPC method to characterization of branched polymers, we need a knowledge on the intrinsic viscosity-molecular weight relationship of branched polymers, either experimental or theoretical. On the other hand, Tung has proposed a new characterization method of branched polymers which is based on GPC and sedimentation velocity measurements. ${ }^{5,6}$ In this method, no knowledge of the viscositymolecular weight relationship is required, but the above-mentioned difficulties concerning GPC measurements are not done away with.

In our previous paper, ${ }^{7}$ we proposed a charac- terization method for obtaining the molecular weight distribution and the branching parameter from sedimentation velocity and diffusion measurements, and reported the results of its preliminary test. Although our method is based on some theoretical assumptions, the experimental procedures required are not laborious compared with those required in the existing methods, and the molecular weight distribution obtained were compatible with the polymerization mechanism of the test samples. However, the previous test ${ }^{7}$ was limited only to two samples of randomly branched polymers prepared by the radical mechanism.

Thus, we here report the results of more extensive test of our method. The relationship between molecular weight and sedimentation constant and that between molecular weight and diffusion constant are derived for each test sample of branched polystyrene and they are analysed to evaluate the average density of a branch point or branching parameter using the 
Kurata-Fukatsu theory of sedimentation constant. $^{8,9}$

\section{OUTLINE OF METHOD ${ }^{7}$}

The molecular weight distribution of linear polymers can be obtained from either sedimentation velocity or diffusion measurements at the theta point, provided that the sedimentation constant $v s$. molecular weight relationship or the diffusion constant $v s$. molecular weight relationship for monodisperse samples is available: ${ }^{10,11}$

$$
\begin{aligned}
& S=K_{S \theta} M^{0.5} \\
& D=K_{D \theta} M^{-0.5}
\end{aligned}
$$

Here $S$ and $D$ are the sedimentation constant and the diffusion constant at the theta point, respectively, $K_{S \theta}$ and $K_{D \theta}$ are constants characteristic of the polymer-theta solvent system, and $M$ is the molecular weight. The distribution curve of $S$ or $D$ can be readily converted into the molecular weight distribution curve $W(M)$ with the aid of eq 1 or 2 .

In the case of branched polymers, the formal application of the above procedure yields the distribution curve of apparent molecular weight $M_{S}$ or $M_{D}$, instead of the real molecular weight $M$. The sedimentation and diffusion constants are affected by the type and density of branching, and eq 1 and 2 are now to be modified as ${ }^{9}$

$$
\begin{aligned}
& S=K_{S \theta} M_{S}^{0.5}=h^{-1} K_{S \theta} M^{0.5} \\
& D=K_{D \theta} M_{D}^{-0.5}=h^{-1} K_{D \theta}{ }^{-0.5}
\end{aligned}
$$

provided that the partial specific volume of the polymer in the solution is not changed by chain branching. The branching parameter $h$, which is a function of branch-point density and $M$, represents the contraction of effective hydrodynamic radius of a polymer molecule due to branching. ${ }^{12}$ Thus, elimination of $h$ from eq 3 and 4 yields

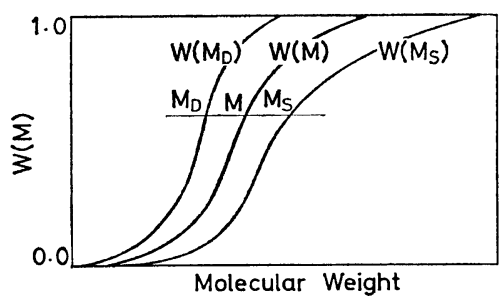

Figure 1. Calculation method for molecular weight distribution curve (MWDC) of branched polymer: $W\left(M_{D}\right)$, apparent MWDC obtained from diffusion method; $W\left(M_{S}\right)$, apparent MWDC obtained from sedimentation velocity method; $W(M)$, real MWDC calculated by $M=\left(M_{D} \cdot M_{S}\right)^{0.5}$.

$$
M=\left(M_{S} \cdot M_{D}\right)^{0.5}
$$

And, if the sedimentation velocity and diffusion measurements are both carried out on a branched polymer sample, and if two constants $K_{S \theta}$ and $K_{D \theta}$ are available, we can derive the distribution curve of real molecular weight, $W(M)$, from two apparent molecular weight distributions $W\left(M_{S}\right)$ and $W\left(M_{D}\right)$ by using eq 5 . As schematically shown in Figure 1, the use of eq 5 should be made at the same integral points on the apparent distribution curves, $W\left(M_{S}\right)$ and $W\left(M_{D}\right)$.

In addition, elimination of $M$ from eq 3 and 4 gives

$$
h^{2}=\left(M_{D} / M_{S}\right)^{0.5}
$$

which enables us to estimate the parameter $h$ at each given integral point of the molecular weight distribution curve. The relationship between $h$ and $M$ thus determined provides useful information on the type of branching.

\section{EXPERIMENTAL}

\section{Materials}

Three samples of styrene-divinylbenzene copolymers were prepared by the same method

Table I. Polymerization conditions of branched polymers

\begin{tabular}{ccccccc}
\hline Sample & $\begin{array}{c}\text { Styrene, } \\
\mathrm{cc}\end{array}$ & $\begin{array}{c}\text { Divinylbenzene, } \\
\mathrm{cc}\end{array}$ & $\begin{array}{c}\mathrm{AIBN},{ }^{\mathrm{a}} \\
\mathrm{g}\end{array}$ & $\begin{array}{c}\text { Temp, } \\
{ }^{\circ} \mathrm{C}\end{array}$ & $\begin{array}{c}\text { Time, } \\
\mathrm{hr}\end{array}$ & $\begin{array}{c}\text { Conversion, } \\
\%\end{array}$ \\
\hline PSB-3 & 30 & 0.027 & 0.033 & 70 & 7.0 & 26 \\
PSB-4 & 30 & 0.028 & 0.038 & 70 & 6.0 & 25 \\
PSB-5 & 60 & 0.028 & 0.024 & 70 & 7.5 & 21 \\
\hline
\end{tabular}

a AIBN, azobisisobutyronitrile. 
Table II. Values of molecular weights and branching parameters

\begin{tabular}{|c|c|c|c|c|c|c|}
\hline \multirow{3}{*}{ Sample } & \multicolumn{4}{|c|}{ Molecular weight } & \multicolumn{2}{|c|}{ Branching parameter } \\
\hline & \multicolumn{2}{|c|}{ Calculated } & \multicolumn{2}{|c|}{ Observed } & \multirow{2}{*}{$\begin{array}{c}\text { Calculated } \\
\frac{\sum h_{i}{ }^{3} M_{i}^{0.5} w_{i}}{\sum M_{i}^{0.5} w_{i}}\end{array}$} & \multirow{2}{*}{$\begin{array}{l}\text { Observed } \\
{[\eta]_{b \theta} /[\eta]_{1} \theta^{\mathrm{b}}}\end{array}$} \\
\hline & $M_{w} \times 10^{-4}$ & $M_{n} \times 10^{-4}$ & $M_{w} \times 10^{-4}$ & $M_{n} \times 10^{-4}$ & & \\
\hline PSB-3 & 26.2 & 12.0 & 28.0 & 14.2 & $\begin{array}{l}0.72 \\
(0.76)^{a}\end{array}$ & 0.78 \\
\hline PSB-4 & 21.4 & 9.7 & 22.4 & 8.4 & $\begin{array}{l}0.66 \\
(0.75)^{\mathrm{a}}\end{array}$ & 0.64 \\
\hline PSB-5 & 38.8 & 26.6 & 39.5 & 27.4 & $\begin{array}{l}0.79 \\
(0.77)^{\mathrm{a}}\end{array}$ & 0.79 \\
\hline
\end{tabular}

described in our previous paper. ${ }^{7}$ The polymerization conditions employed are shown in Table I.

\section{Solvents}

Cyclohexane and methylethylketone (MEK) were purified by the ordinary methods. Cyclohexane was used in sedimentation velocity, diffusion and intrinsic viscosity measurements and MEK was used in light scattering and osmotic pressure measurements as solvents.

\section{Light Scattering}

Light scattering measurements were carried out in MEK at $35^{\circ} \mathrm{C}$ for a light of $436 \mathrm{~nm}$ by using a Shimazu PG-21 type light scattering photometer with a cylindrical cell. The observed refractive index increment was $0.240 \mathrm{ml} / \mathrm{g}$ at $35^{\circ} \mathrm{C}$, which agreed with $0.235 \mathrm{ml} / \mathrm{g}$ for randomly branched polystyrene in MEK at $35^{\circ} \mathrm{C}$ for $436 \mathrm{~nm}$ reported by Kurata, et al. ${ }^{9}$ The difference between these observed values is considered to be within experimental error. The value of the refractive index of MEK was 1.388 at $436 \mathrm{~nm}^{13}$ The weight average molecular weights $M_{w}$, calculated by the Zimm plot, are shown in Table II.

\section{Osmotic Pressure}

Osmotic pressure measurements were carried out in MEK at $35 \pm 0.001^{\circ} \mathrm{C}$ by a modified Zimm-Myerson type osmometer with a gel cellophane membrane. The dynamic osmotic method was employed in the measurements. The number average molecular weights $M_{n}$ obtained are shown in Table II.

\section{Sedimentation Velocity ${ }^{10}$}

Sedimentation velocity measurements were carried out at $35^{\circ} \mathrm{C}$ in cyclohexane by using a Hitachi Ultracentrifuge with Schlieren optics. The measurements were made at $43,700 \mathrm{rpm}$ and at four concentrations. Apparent molecular weight distribution curves $W\left(M_{S}\right)$ were obtained by using the standard method for corrections of diffusion, concentration and pressure effects. ${ }^{14}$ As stated above our method is based on the assumption that the partial specific volume of branched polymer is equal to that of the linear one. To test the validity of this assumption, we carried out density measurements on polymer solutions in cyclohexane at $35 \pm 0.01^{\circ} \mathrm{C}$ by using an Ostowald densitometer. The results are shown in Figure 2. The partial specific volume $\bar{v}$ of polymer in dilute solution can be calculated from the slope of the straight line in Figure 2 which corresponds to the quantity

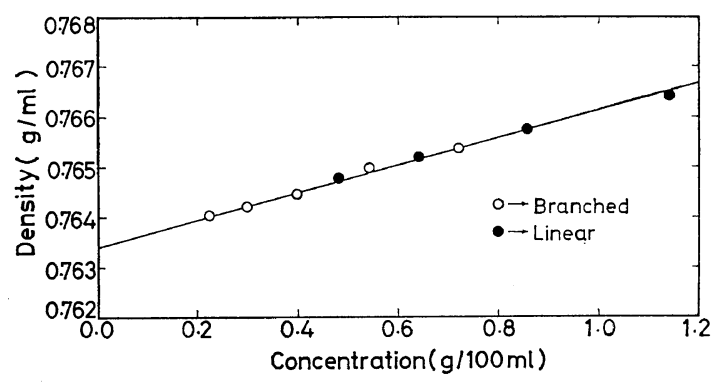

Figure 2. Determination of the partial specific volumes $\bar{v}$ of linear and branched polystyrene in cyclohexane at $35^{\circ} \mathrm{C}: \bar{v}=0.934 \mathrm{ml} / \mathrm{g}$ for the both samples. 
$\left(1-\bar{v} \rho_{0}\right)$, where $\rho_{0}$ is the density of the solvent. ${ }^{15}$ The observed value of $\bar{v}$ were $0.934 \mathrm{ml} / \mathrm{g}$ for both the linear and the branched polystyrenes. This value is in good agreement with $0.934 \mathrm{ml} / \mathrm{g}$ reported by Meyerhoff ${ }^{16}$ and $0.933 \mathrm{ml} / \mathrm{g}$ by Kurata, et al. ${ }^{9}$ for linear polystyrenes.

\section{Diffusion ${ }^{11}$}

Diffusion measurements were carried out in cyclohexane at $35^{\circ} \mathrm{C}$ using a Hitachi HTB-type electrophoresis apparatus with Schlieren optics. A new type diffusion cell, designed by Claesson, et al. ${ }^{17}$ and constructed in our laboratory, ${ }^{18}$ was used. The diffusion constants were calculated by the moment method and the area method, and extrapolated to infinite dilution. The weight distribution curves of diffusion constant were determined by assuming Gralén's distribution function applies for the molecular weight. ${ }^{19}$

\section{Viscosity}

Viscosities were measured using a dilution viscometer of the Ubbelohde type. All measurements were made in cyclohexane at $35^{\circ} \mathrm{C}$. The intrinsic viscosities were determined from plots of $\eta_{\mathrm{sp}} / C$ vs. $C$, where $\eta_{\mathrm{sp}}$ and $C$ denote the specific viscosity and concentration in $\mathrm{g} / 100 \mathrm{~m} l$, respectively. The intrinsic viscosity of a linear polymer having the same molecular weight as that of branched one was calculated by the equation ${ }^{20}$

$$
[\eta]=7.48 \times 10^{-4} M^{0.5}, \quad(100 \mathrm{ml} / \mathrm{g})
$$

\section{RESULTS AND DISCUSSIONS}

In the previous paper, ${ }^{7}$ we showed that the $\theta$ temperature for branched polystyrene was $35^{\circ} \mathrm{C}$ in cyclohexane which was equal to that of linear polystyrene. Then, we found that the partial specific volume of branched polystyrene in cyclohexane at $35^{\circ} \mathrm{C}$ was $0.934 \mathrm{ml} / \mathrm{g}$, which was equal to that of the linear one observed in this work and reported also by Meyerhoff ${ }^{16}$ and Kurata, et al. ${ }^{9}$ Therefore, use of our method is justified, at least in principle, for determination of the molecular weight distribution and the branching parameter of branched polymers.

\section{Molecular Weight Distribution}

Examples of the weight distribution curve of sedimentation constants $W(S)$ and that of dif-

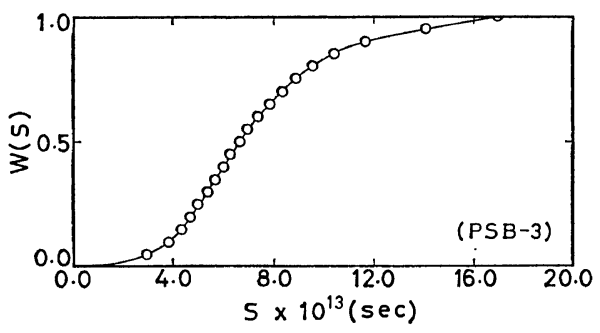

Figure 3. Integral weight distribution curve of sedimentation constant $S$ for PSB-3.

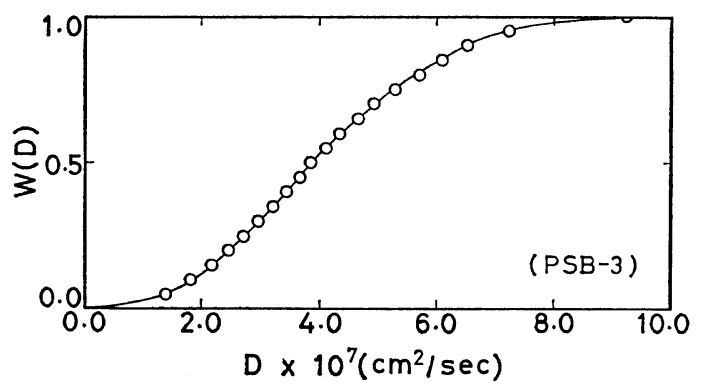

Figure 4. Integral weight distribution curve of diffusion constant $D$ for PSB-3.

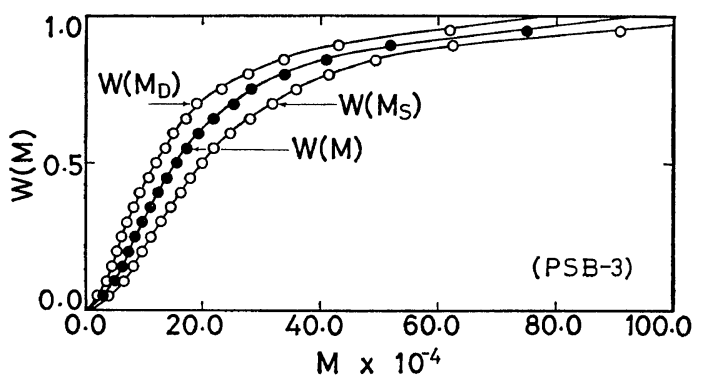

Figure 5. Integral weight distribution curves for molecular weight for PSB-3.

fusion constant $W(D)$ are shown in Figures 3 and 4 , respectively. The apparent molecular weight distribution curve $W\left(M_{S}\right)$ and $W\left(M_{D}\right)$ shown in Figure 5 were obtained from the curves given in Figures 3 and 4 by using the equations $\mathrm{s}^{21,22}$

$$
\begin{gathered}
S=1.48 \times 10^{-15} M^{0.5} \quad(\mathrm{sec}) \\
D=1.43 \times 10^{-4} M^{-0.5} \quad\left(\mathrm{~cm}^{2} / \mathrm{sec}\right)
\end{gathered}
$$

The real molecular weight distribution curve $W(M)$ obtained by eq 5 is also shown in Figure 5. In our method, it is essential to determine 
correctly the constant values $K_{S \theta}$ and $K_{D \theta}$ in eq 1 and 2. The substitution of eq 1 and 2 into the Svedberg equation yields

$$
R T\left(K_{S \theta} / K_{D \theta}\right) /\left(1-\bar{v} \rho_{0}\right)=1
$$

where $R$ is the gas constant, $T$ the absolute temperature, $\bar{v}$ the partial specific volume of linear polymer in solution and $\rho_{0}$ the density of solvent. Putting $R=8.314 \times 10^{7} \mathrm{erg} \cdot \mathrm{mol}^{-1} \mathrm{deg}^{-1}$, $T=308.15 \mathrm{deg}, \rho_{0}=0.7634 \mathrm{~g} / \mathrm{cm}^{3}, \bar{v}=0.934 \mathrm{~cm}^{3} / \mathrm{g}$, $K_{S \theta}=1.48 \times 10^{-15} \mathrm{sec}(\mathrm{mol} / \mathrm{g})^{0.5}$ and $K_{D \theta}=1.43 \times$ $10^{-4}\left(\mathrm{~cm}^{2} / \mathrm{sec}\right)(\mathrm{g} / \mathrm{mol})^{0.5}$, we obtain 0.924 for the left-hand side of eq 10; the deviation of this value from unity seems to be within experimental error. According to Fujita, ${ }^{23}$ the literature values of $K_{S \theta}$ for polystyrene-cyclohexane system at $35^{\circ} \mathrm{C}$ scatter around $1.45 \times$ $10^{-15} \mathrm{sec}(\mathrm{mol} / \mathrm{g})^{0.5}$. From these figures, the combining use of eq 8 and 9 , obtained by different authors, may be justified experimentally. The average molecular weights, calculated from the molecular weight distribution curves, are shown in Table II with the values obtained by osmotic pressure and light scattering measurments. The calculated values are in good agreement with those directly observed. In our method, the distribution curve of the diffusion constant was calculated by assuming the distribution function of Gralén's type to be applicable. ${ }^{19}$ It was reported in our previous paper that the molecular weight distribution of linear polystyrenes, polymerized by radical mechanism, could be expressed to a good approximation by the Gralén function. ${ }^{24}$ Since the present samples of branched polystyrene were also polymerized by radical mechanism, use of the Gralén distribution function may be justified at least from a practical standpoint. However, this will be come a weak point for the present study, since the shape of molecular weight distribution may be considerably affected by chain branching. To eliminate uncertainty concerning this assumption, some independent tests of the molecular weight distribution is to be carried out in future.

\section{Branching Parameter}

According to eq 6 and Figure 1, the branching parameter $h$ is expressed as a function of molecular weight. An example of the relation

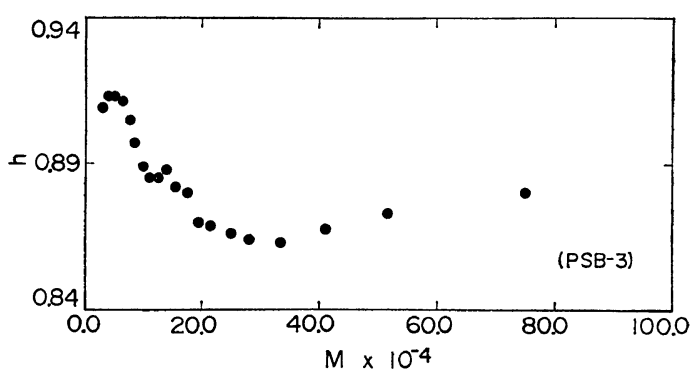

Figure 6. Relation between molecular weight $M$ and branching parameter $h$ for PSB-3.

between branching parameter and molecular weight is shown in Figure 6 . If the branching parameter $h$ decreases with molecular weight monotonously, the distribution curve of branching parameter will be obtained. As seen in Figure 6, however, the monotoneous variation was not observed and so the distribution curve of branching parameter was not obtained. In order to compare the average values of branching parameter, calculated using by Figure 6 and molecular weight distribution curve, with the viscosity data $[\eta]_{b \theta} /[\eta]_{1 \theta}$, the following method was applied: where $[\eta]_{b \theta}$ and $[\eta]_{1 \theta}$ are intrinsic viscosities of branched polymer and linear one having the same molecular weight. According to Stockmayer and Fixman, the intrinsic viscosity of branched polymer at the $\theta$ temperature is given by the equation ${ }^{12}$

$$
[\eta]_{b \theta}=h^{3} K_{\theta} M^{0.5}
$$

where $h$ is the branching parameter and $K_{\theta}$ is a constant. Taking into account the fact that intrinsic viscosity is expressed by a weight average value, we have

$$
[\eta]_{b \theta} /[\eta]_{1 \theta}=\sum_{i} h_{i}{ }^{3} M_{i}^{0.5} w_{i} / \sum_{i} M_{i}^{0.5} w_{i}
$$

where $h_{i}$ and $w_{i}$ are the branching parameter and the weight fraction of the molecular species with molecular weight $M_{i}$. If the average value of the right-hand side of eq 12 is calculated by using molecular weight distribution curve and Figure 6, the average values may correspond to those of $[\eta]_{b \theta} /[\eta]_{1 \theta}$. The caluculated values are shown in Table II. In evaluation of the lefthand side of eq 12, $[\eta]_{b \theta}$ is measured as in the Experimental section. The intrinsic viscosity 
$[\eta]_{1 \theta}$ is calculated by eq 7 , where the weight average molecular weight obtained by light scattering measurements is used for $M$. In Table II, the values of $[\eta]_{b \theta} /[\eta]_{1 \theta}$ thus obtained are tabulated as the observed values of branching parameter. These calculated values are in good agreement with those observed within the range of experimental error. In our previous paper, ${ }^{7}$ $[\eta]_{b \theta} /[\eta]_{1 \theta}$ was approximated by $\left(\sum_{i} w_{i} h_{i} / \sum_{i} w_{i}\right)^{3}$. Although the approximation is not so accurate, it seems that the numerical values do not significantly vary owing to the narrow distribution of branching parameter of the samples.

Molecular Weight Dependences of Sedimentation and Diffusion Constants

One of the objects for the present study is to establish the molecular weight dependences of solution properties such as sedimentation constant and diffusion constant for the monodisperse branched polymers. Kamada, et al., ${ }^{25,26}$ and Kurata, et al., ${ }^{9}$ studied some solution properties of branched polymers, but the discussion of their results did not include any correction for polydispersity. In order to obtain the relationship between molecular weight and the sedimentation constant for monodisperse branched polymers, the following method was applied. If the values for the molecular weight $M_{i}$ and sedimentation constant $S_{i}$ at the same integral values on each distribution curve in Figures 3 and 5 are recorded, the curve of $\log S$ vs. $\log M$ may be obtained for the monodisperse branched polymers, because $M_{i}$ and $S_{i}$ perhaps belong to the same molecular species $i$. Similarly the relation between $\log D$ and $\log M$ is obtained. They are shown in Figures 7 and 8, respectively, together with the relation for linear polystyrene in cyclohexane at $35^{\circ} \mathrm{C}(h=1)$. The lines for experimental points were drawn using the least squares method. It is seen in Figures 7 and 8 that the values of $S$ and $D$ deviate from those of linear polymer. The curve in Figure 7 behaves like those obtained by Kamada, et al., and Kurata, et al.

According to the Kurata-Fukatsu theory for randomly branched polymers with tetra-functional branch units, ${ }^{8}$ sedimentation constant is approximated as $^{9}$

$$
S=K_{S \theta} h^{-1} M^{0.5}
$$

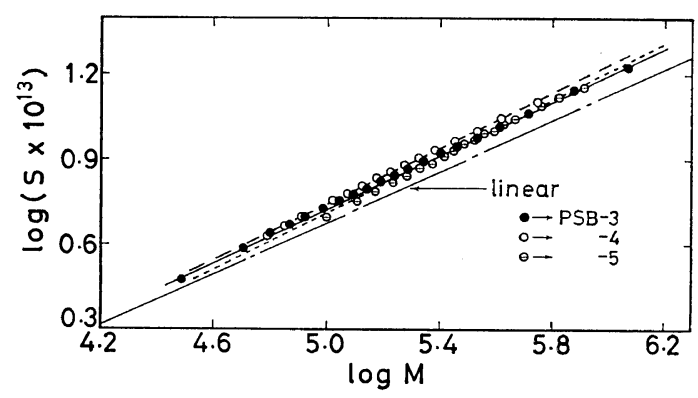

Figure 7. The relationships between $\log S$ and $\log M$ for branched samples.

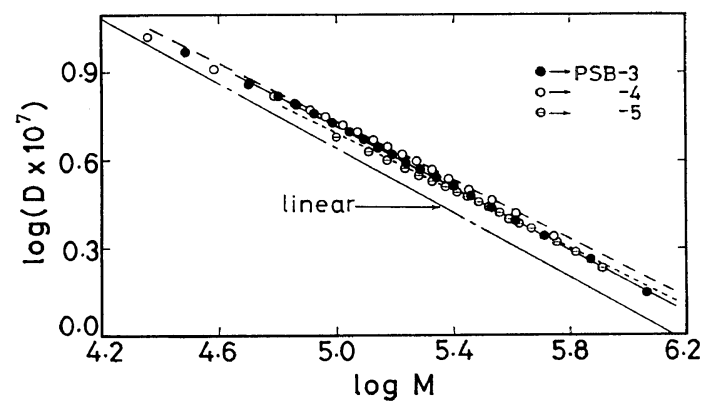

Figure 8. The relationships between $\log D$ and $\log M$ for branched samples.

$$
h^{-1}=0.605(\lambda M)^{1 / 4}+0.474(\lambda M)^{-1 / 4}
$$

where $\lambda$ is the branch point density expressed by the number of branch units per monomer. Similarly we have

$$
D=K_{D \theta} h^{-1} M^{-0.5}
$$

If $\lambda$ in eq 14 is a constant for each sample, eq 13 and 15 give to the equations

$$
\begin{aligned}
& S M^{-1 / 4}=0.605 K_{S \theta} \lambda^{1 / 4} M^{1 / 2}+0.474 K_{S \theta} \lambda^{-1 / 4} \\
& D M^{3 / 4}=0.605 K_{D \theta} \lambda^{1 / 4} M^{1 / 2}+0.474 K_{D \theta} \lambda^{-1 / 4}
\end{aligned}
$$

If $S M^{-1 / 4}$ and $D M^{3 / 4}$ are plotted against $M^{1 / 2}$, we should have the straight lines as shown in Figures 9 and 10, respectively. The slope and the intercept give the branch point density $\lambda$. In Figures 9 and 10, the lines are drawn so as to give the same values of $\lambda$ from the intercept and the slope. As is seen in eq 14 the theory is not valid for the limit of linear polymer $(h=1$ and $\lambda=0)$. The plots for linear polymer, therefore, cannot be shown in Figures 9 and 10. The average values of branch point 
density, $\lambda$, obtained from Figures 9 and 10 are shown in Table III. The values are comparable to those obtained by Kurata, et al., for randomly branched polystyrene. ${ }^{9}$ In the figures, the plots obtained by experiments deviate from the straight lines at the higher and lower molecular weight regions. In the higher molecular weight region, the deviation may be said to result from the experimental error in sedimentation velocity and diffusion measurements. The causes of the deviation in the low molecular weight region may be the experimental error and the fact that the Kurata-Fukatsu theory can not be applicable to the low molecular weight region. However, if the values of branch point density $\lambda$ depend upon the molecular weights, the curves also deviate from the straight lines. In such a case, eq 16 and 17 cannot be expected to hold. Therefore, the deviations from the straight lines in Figures 9 and 10 are considered to be due to experimental error and inadequacy of the

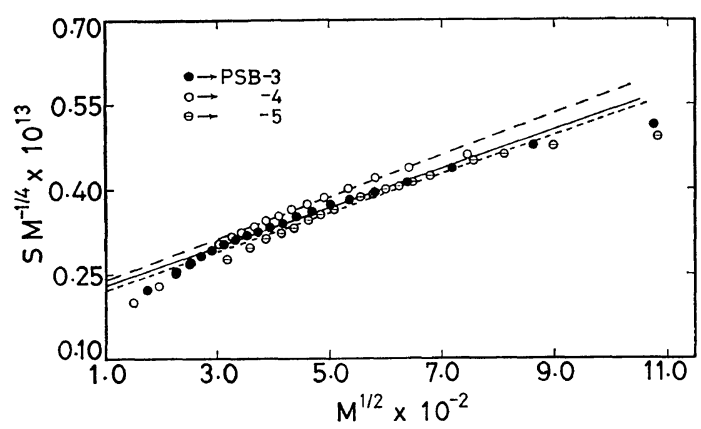

Figure 9. Plots of $S M^{-1 / 4}$ vs. $M^{1 / 2}$ according to eq 16.

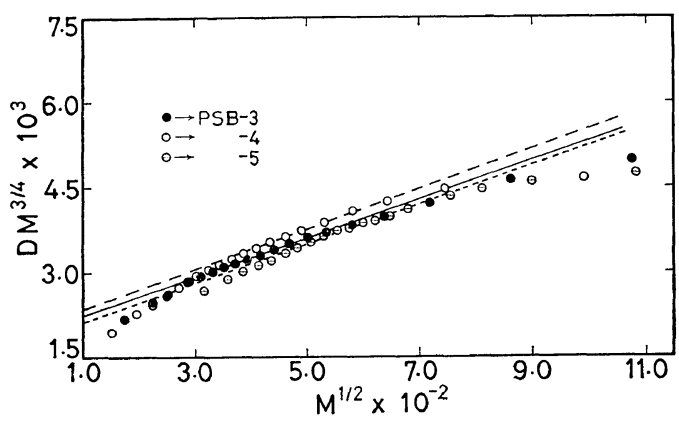

Figure 10. Plots of $D M^{3 / 4}$ vs. $M^{1 / 2}$ according to eq 17.
Table III. Branching densities

$\begin{array}{cc}\text { Sample } & \begin{array}{c}\text { Branching density } \\ \lambda \times 10^{5}\end{array} \\ & 0.23 \\ \text { PSB-3 } & 0.21 \\ \text { PSB-4 } & 0.26 \\ \text { PSB-5 } & \end{array}$

theory. In any case, Figures 9 and 10 are the experimental tests of the Kurata-Fukatsu theory, and the theory may be a good approximation for randomly branched polymers at the present stage.

Using the values of $\lambda$, eq 14 and molecular weight distribution curves, the values of $\sum_{i} h_{i}{ }^{3} M_{i}^{0.5} w_{i} / \sum_{i} M_{i}^{0.5} w_{i}$ were calculated. These values are shown in parentheses in Table II. These values, calculated by our method, showed a slight deviation from those determined using the Kurata-Fukatsu theory, but both are comparable with viscosity data $[\eta]_{b \theta} /[\eta]_{1 \theta}$. In conclusion, sedimentation and diffusion measurements provide useful informations on chain branching.

\section{Practical Limitations of Our Method}

Taking into account the fact that the molecular weight distribution function should be assumed as analytical for obtaining molecular weight distribution curve by the diffusion method, the practical limitations of our method are discussed. As the diffusion method cannot in general be applied to a sample with two peaks in the differential distribution curve of molecular weight, neither can our method be applied to such a sample. This method, therefore, should not be applied without considering the polymerization conditions of samples. Gralén's distribution function has been assumed in the present study. Moreover, even if the sample should have one peak in the molecular weight distribution curve, the application of our method may be limited in connection with the value of branching parameter as follows: Consider the two monodisperse molecular species with molecular weights $M_{i}$ and $M_{j}$ and branching parameters $h_{i}$ and $h_{j}$, respectively, in a polydisperse sample. Assume that the apparent and real molecular weight distribution curves $W\left(M_{S}\right), W\left(M_{D}\right)$ and $W(M)$ are represented as 
shown in Figure 1. Now, if $M_{j}>M_{i}$, the following relations may be found to hold:

$$
\begin{aligned}
& M_{S j}>M_{S i} \\
& M_{D j}>M_{D i}
\end{aligned}
$$

Here, $M_{S i}, M_{S j}, M_{D i}$, and $M_{D j}$ are the apparent molecular weights obtained by eq 3 and 4 for molecular species $i$ and $j$, respectively. If the branching degree increases with molecular weight $\left(h_{i}>h_{j}\right)$, eq 5 and 6 give the relations

$$
\begin{aligned}
& M_{j} / M_{i}=\left(M_{D j} M_{S j} / M_{D i} M_{S i}\right)^{0.5}>1 \\
& \left(h_{i} / h_{j}\right)^{2}=\left(M_{D i} M_{S j} / M_{D j} M_{S i}\right)^{0.5}>1
\end{aligned}
$$

Since relations 18 and 19 must be satisfied in relations 20 and 21 , the following relation is needed $^{7}$

$$
M_{j} / M_{i}>\left(h_{i} / h_{j}\right)^{2}>1
$$

Analogously, if the branching degree decreases with molecular weight $\left(h_{j}>h_{i}\right)$, the other relation is obtained as

$$
M_{j} / M_{i}>\left(h_{j} / h_{i}\right)^{2}>1
$$

Therefore our method is applicable to a branched polymer which has one peak in the molecular weight distribution curve and satisfies relations 22 or 23 . Moreover, our method can be applied to the sample that $h$ is constant. The relations 22 and 23 may imply that the branching parameter distribution of a sample must be narrower than the distribution of molecular weight. However, we cannot determine the limits of numerical values of relations 22 and 23 , and this problem is left for further study. Samples, polymerized by ordinary radical mechanism, may satisfy relation 22 . Therefore, in Figures 7 and 8 , the deviations of the curves from the lines of linear polymer in the higher molecular weight regions would be understandable.

From our experimental results, it may be concluded that although our method contains some assumptions and limitations, fairy good information of molecular weight distribution and branching parameter may be obtained for randomly branched polymers.

Acknowledgements. We would like to express our sincere appreciation to Professors Takahide
Saito and Naomichi Iso of Tokyo University of Fisheries for granting permission to use the Ultracentrifuge at their laboratory. Gratitude is due also to Professor Taiichi Shibuya of the Faculty of Textile Science, Shinshu University for reading and correcting this manuscript.

\section{REFERENCES}

1. Z. Grubisic, P. Rempp, and H. Benoit, $J$. Polym. Sci., Part B, 5, 753 (1967).

2. E. E. Drott and R. A. Mendelson, ibid., Part $A-2,8,1361$ (1970).

3. E. E. Drott and R. A. Mendelson, ibid., Part $A-2,8,1375$ (1970).

4. M. Kurata, H. Okamoto, M. Iwama, M. Abe, and T. Homma, Polym. J., 3, 739 (1972).

5. L. H. Tung, J. Polym. Sci., Part A-2, 7, 47 (1969).

6. L. H. Tung, ibid., Part A-2, 9, 759 (1971).

7. H. Matsuda, I. Yamada, and S. Kuroiwa, Polym. J., 8, 415 (1976).

8. M. Kurata and M. Fukatsu, J. Chem. Phys., 41, 2934 (1964).

9. M. Kurata, M. Abe, M. Iwama, and M. Matsushima, Polym. J., 3, 729 (1972).

10. H. W. McComic, "Sedimentation," in "Polymer Fractionation," M. J. R. Cantow, Ed., Academic Press, New York, N.Y., 1967, p 251.

11. W. Buchard and H.-J. Cantow, "Isothermal Diffusion," in "Polymer Fractionation," M. J. R. Cantow, Ed., Academic Press, New York, N.Y., 1967, p 285.

12. W. H. Stockmayer and M. Fixman, Ann. N.Y. Acad. Sci., 57, 334 (1953).

13. P. Doty and R. F. Steiner, J. Chem. Phys., 18, 1211 (1950).

14. H. Fujita, "Foundations of Ultracentrifugal Analysis," Wiley-Interscience, New York, N.Y., 1975, p 127.

15. H. Fujita, T. Kotaka, and H. Utiyama, "Method of Ultracentrifugal Analysis," in "Zoku Seibutsubutsurigaku Koza (Second Series of Biophysics)," Vol. II, J. Tanaka and A. Wada, Ed., Yoshioka Shoten, Kyoto, 1968, p 147.

16. G. Meyerhoff, "Problems in the Determination of Molecular Weight Distribution by Sedimentation Transport," in "Ultracentrifugal Analysis in Theory and Experiment," J.W. Williams, Ed., Academic Press, New York, N.Y., 1963, p 51.

17. S. Claesson, H. Matsuda, and L.-O. Sundelöf, Chemica Scripta, 6, 94 (1974). 
18. H. Matsuda, M. Okabe, and S. Kuroiwa, Kobunshi Ronbunshu, 33, 116 (1976).

19. N. Gralén, Kolloid-Z., 95, 188 (1941).

20. H. Matsuda, M. Takeshima, S. Shimizu, and S. Kuroiwa, Makromol. Chem., 134, 309 (1970).

21. L. H. Tung and J. R. Runyon, J. Appl. Polym. Sci., 17, 1589 (1973).

22. A. Kotera, T. Saito, H. Matsuda, and K. Takemura, Rept. Progr. Polym. Phys. Jpn., 14,
39 (1971).

23. H. Fujita, "Foundations of Ultracentrifugal Analysis," Wiley-Interscience, New York, N.Y., 1975, p 206.

24. N. Yamada and H. Matsuda, Kobunshi Kagaku (Chem. High Polymers), 18, 114 (1961).

25. K. Kamada and H. Sato, Polym. J., 2, 489 (1971).

26. K. Kamada and H. Sato, ibid., 2, 593 (1971). 